\title{
A Comparative Study of Bronchoscopic Microsample Probe versus Bronchoalveolar Lavage in Patients with Burns-Related Inhalational Injury, Acute Lung Injury and Chronic Stable Lung Disease
}

\author{
Suveer Singh ${ }^{a, b}, d$ Vimal Grover ${ }^{b, d, e}$ Linsey Christie ${ }^{b}$ Peter Charles $^{g}$ \\ Peter Kelleher ${ }^{c, e, g}$ Pallav L. Shah ${ }^{a, f}$ \\ ${ }^{a}$ Department of Respiratory Medicine, ${ }^{\mathrm{b}}$ Magill Department of Critical Care, Anaesthesia and Pain and ${ }^{\mathrm{c}}$ Directorate of \\ HIV and Sexual Health, Chelsea and Westminster NHS Foundation Trust, ${ }^{\mathrm{d}}$ Division of Surgery and Cancer, ${ }^{\mathrm{e}}$ Immunology \\ Section, Division of Infectious Diseases and ${ }^{\mathrm{f}}$ National Heart and Lung Institute, Imperial College London, and ${ }^{9}$ Division \\ of Immunology, Imperial College Healthcare NHS Trust, London, UK
}

\section{Key Words \\ Bronchoscopic microsample probe $\cdot$ Bronchoalveolar lavage $\cdot$ Burns-related inhalational injury $\cdot$ Acute lung injury $\cdot$ Chronic obstructive lung disease $\cdot$ Interstitial lung disease}

\begin{abstract}
Background and Objectives: The bronchoscopic microsample (BMS) probe allows direct epithelial lining fluid (ELF) level measurement without saline lavage. We investigated whether cytokine levels in ELF from a BMS differed from those obtained by bronchoalveolar lavage (BAL) in stable and acute lung disease. Methods: In a single-centre, prospective observational cohort study of 45 patients, a sequential BMS probe procedure and BAL were performed on patients with stable chronic obstructive lung disease, interstitial lung disease, acute lung injury (ALI), burns-related inhalational injury or controls. ELF samples were assayed for IL-1 $\beta$, IL-6, IL-8, TNF- $\alpha$ and G-CSF. Results: Both bronchoscopic microsampling and BAL showed significantly higher cytokine levels in the ELF from patients with ALI and burnsrelated inhalational injury than from those with chronic stable lung disease. The BMS method detected cytokine levels
\end{abstract}

approximately 20- to 80-fold higher than the corresponding BAL (uncorrected for dilution). The ratio of BMS and BAL cytokine levels was as follows: the ratio for IL-1 $\beta$ [mean $55,95 \%$ confidence interval (CI) 34-88] was higher than that for IL-6 (mean 16, 95\% Cl 10-23, $\mathrm{p}=0.015$ ) and IL-8 (mean 13, 95\% $\mathrm{Cl}-5$ to $36, \mathrm{p}=0.03$ ). The ratio for $\mathrm{G}-\mathrm{CSF}$ (mean $43,95 \% \mathrm{Cl}$ 24-75) was higher than that for IL-6 (mean $16,95 \%$ Cl 10-23, $p=0.008$ ). Conclusions: The BMS probe safely collects ELF with higher equivalent inflammatory cytokine concentrations than via BAL from patients with both acute and chronic lung disease and can be an alternative to saline BAL. Variations in cytokine concentrations between BMS and BAL and sampling-site differences warrant further study.

(c) 2015 S. Karger AG, Basel

\section{Introduction}

The use of bronchoalveolar lavage (BAL) to investigate inflammatory biomarkers and microbiological organisms within the lung in respiratory and critically ill patients is well established. It requires a significant volume load of saline administered to a pulmonary sub-segment, with variable fluid retrieval and potential hypoxia in me-

\section{KARGER 125}

C 2015 S. Karger AG, Base

$0025-7931 / 15 / 0891-0019 \$ 39.50 / 0$

E-Mail karger@karger.com

www.karger.com/res
Dr. Suveer Singh, BSc, MBBS, PhD, EDIC, DICM, FFICM, FRCP

Consultant Respiratory and Intensive Care Physician

Department of Respiratory Medicine, Chelsea and Westminster Hospital

369 Fulham Road, London SW10 9NH (UK)

E-Mail suveer.singh@imperial.ac.uk 
chanically ventilated patients or those with chronic obstructive lung disease (COPD). Furthermore, soluble protein analysis of BAL fluid suffers from inaccuracies related to saline dilution [1].

A bronchoscopic microsample (BMS) probe has been described which allows absolute cytokine levels in the epithelial lining fluid (ELF) to be measured directly without lavage [2]. It comprises a polyethylene outer sheath (1.7 $\mathrm{mm}$ in diameter) and an inner fibre rod probe (1.2 $\mathrm{mm}$ in diameter and $30 \mathrm{~mm}$ in length) attached to a stainless steel guide wire that is $10 \mathrm{~cm}$ long. It has been employed in the sampling of biomarkers in acute lung injury (ALI) $[2,3]$ and the measurement of pulmonary drug concentrations $[4,5]$ as well as an adjunctive diagnostic sampling modality in suspected lung cancer [6]. As of yet, a comparison with BAL of its utility for biomarker sampling has not been performed in patients with COPD or in burns-related inhalational injury, two areas of active biomarker research. We compared inflammatory cytokine levels from ELF obtained by the BMS probe with those from BAL in controls, in patients with chronic stable lung disease and in mechanically ventilated patients with burns-related inhalational injury or ALI. We investigated whether there was a difference in the biomarker concentrations in ELF from the BMS. We also determined whether the procedure with the BMS probe would be as safe and easy to perform as BAL.

\section{Materials and Methods}

\section{Subjects}

Forty-five patients were recruited. They gave their consent for the study, which took place over 18 months from September 2004. They were all patients who had been admitted to the day-case bronchoscopy clinic, the general intensive care unit (ICU) or the burns service ICU of the Chelsea and Westminster Hospital, Imperial College, London. This is a 500-bed, inner-city academic teaching hospital.

They were recruited into the following 5 groups: (1) 2 with ALI/ acute respiratory distress syndrome who were both mechanically ventilated, (2) 6 with burns-related inhalational injuries, 5 of whom were mechanically ventilated, (3) 15 with COPD (non-ventilated), (4) 18 with interstitial lung disease (ILD, non-ventilated) and (5) 4 non-ventilated control subjects.

The diagnosis of ALI was according to the criteria of the American-European Consensus Conference [7], and that of burnsrelated inhalational injury according to accepted clinical definitions [8]. ALI had been precipitated by bacterial communityacquired pneumonia and septic shock secondary to urosepsis.

The diagnosis of COPD was made using standard clinical criteria and pulmonary function tests [9]. The control and COPD subjects underwent bronchoscopy to identify the cause of haemoptysis, atypical chest pain or to investigate small solitary pe- ripheral pulmonary nodules. Patients with COPD were excluded if chest CT scans revealed suspected parenchymal infiltrate, spiculated mass or airway tumour. According to local multidisciplinary clinic follow-up protocols, no malignancies were identified in any of these patients over 2 years.

The ILD patients comprised 9 with pulmonary sarcoidosis, 6 with idiopathic interstitial pneumonia, 2 with extrinsic allergic alveolitis and 1 with Behçet's disease. Bronchoscopy was performed to assess indicators of potentially steroid responsive disease and to rule out unsuspected chronic infection (including mycobacterial disease). None showed mycobacterial disease when isolated with auramine stain or at 6-weeks of culture.

Anthropometric data were collected. For mechanically ventilated patients, ventilatory parameters, fractional inspired oxygen, partial pressure of arterial oxygen, Acute Physiology and Chronic Health Evaluation (APACHE) II score, blood markers of inflammation/infection (C-reactive protein and white cell count) and microbiological analysis were recorded. For non-ventilated patients, respiratory parameters $\left(\mathrm{FEV}_{1} / \mathrm{FVC}\right)$ were additionally recorded when available.

This study was approved by the Riverside National Research Ethics Review Committee 04/Q0401/62. Written informed consent was obtained from all study participants or assent from a family member or next of kin.

\section{Patient Preparation}

All mechanically ventilated patients were pre-oxygenated and sedated with either midazolam or propofol infusions under standard protocols. No neuromuscular antagonists were required. With regard to the burns patients, sampling was made from mucosal areas visually free of soot. BAL fluid was passed through gauze to remove any particulate soot matter.

For non-ventilated patients in the day-case bronchoscopy clinic, conscious sedation with midazolam (2-5 mg i.v.) was administered according to hospital guidelines with cardio-respiratory monitoring. Topical local anaesthesia ( $2 \%$ lignocaine) was administered to the vocal cords and into the tracheo-bronchial airways prior to sampling. Bronchoscopy was performed by two experienced bronchoscopists (S.S. and P.L.S.).

Adverse events were defined as (1) the need to abandon the procedure due to intolerance, (2) desaturation to $<90 \%$ during the bronchoscopy for $>1 \mathrm{~min}$, and/or adjunctive oxygen $>10$ litres $/ \mathrm{min}$ via the oronasal route, (3) procedure-associated bleeding of $>50 \mathrm{ml}$, (4) a systolic blood pressure of $<90$ or $>160 \mathrm{~mm} \mathrm{Hg}$ during or within the $30 \mathrm{~min}$ after the procedure and (5) a heart rate of $<50 / \mathrm{min}$ and/or $>140 /$ min during or within the $30 \mathrm{~min}$ after the procedure.

\section{Bronchoscopic Microsampling}

Bronchoscopic microsampling and BAL were performed sequentially in all patients as an extension of clinically indicated bronchoscopy. A flexible bronchoscope (BF-IT240; Olympus, Tokyo, Japan) was inserted into the lung (through the endotracheal tube if intubated) for examination of the airway. Excess sputum was suctioned if present and the accessory port was flushed with $10 \mathrm{ml}$ of air to minimize BMS probe contamination. The bronchoscope was advanced to the segmental bronchus of interest. Generally, the right middle-lobe segments (right bronchus RB4 or RB5) were sampled if possible, and for all control groups.

The BMS probe, developed by Ishizaka et al. [2] in conjunction with Olympus (Tokyo, Japan), consists of a 1.7-mm diameter poly- 
Table 1. Characteristics of 45 study patients

\begin{tabular}{|c|c|c|c|c|c|c|c|c|}
\hline Group & $\begin{array}{l}\text { Patients, } \\
\mathrm{n}\end{array}$ & $\begin{array}{l}\text { Age, years } \\
\text { (range) }\end{array}$ & $\begin{array}{l}\text { Males, } \\
\%\end{array}$ & $\begin{array}{l}\text { Smoking status, \% } \\
\text { (current/ex-smoker) }\end{array}$ & $\begin{array}{l}\text { APACHE II } \\
\text { score }^{\mathrm{a}} \text { (range) }\end{array}$ & $\begin{array}{l}\text { Positive } \\
\text { microbiology }\end{array}$ & $\begin{array}{l}\mathrm{FEV}_{1}^{\mathrm{b}}, \text { litres } \\
\text { (range) }\end{array}$ & $\begin{array}{l}\mathrm{FVC}^{\mathrm{b}} \text {, litres } \\
\text { (range) }\end{array}$ \\
\hline Controls & 4 & $35-81$ & 100 & $50 / 25$ & n.a. & none & $1.62-3.77$ & $3.05-4.9$ \\
\hline ILD & 18 & $27-82$ & 56 & $0 / 17$ & n.a. & none & $0.99-4.06$ & $1.93-4.69$ \\
\hline Burns & 6 & $18-84$ & 33 & $33 / 17$ & $12-21$ & 1 MRSA LRTI & n.a. & n.a. \\
\hline
\end{tabular}

E. coli $=$ Escherischia coli LRTI = lower respiratory tract infection; $M$. catarrh = Moraxella catarrhalis; MRSA = methicillin-resistant Staphylococcus aureus; MSSA = methicillin-sensitive S. aureus; n.a. = not available; S. pneumo = Streptococcus pneumoniae; UTI = urinary tract infection.

a Applies to burns and ALI patients only.

${ }^{\mathrm{b}}$ Measured in healthy controls, COPD and ILD patients only.

ethylene outer sheath and an inner fibre rod probe $1.2 \mathrm{~mm}$ in diameter and $30 \mathrm{~mm}$ in length attached to a stainless steel guide wire $10 \mathrm{~cm}$ in length. The inner probe has a protective outer sheath to minimise sample contamination.

The BMS procedure has been described in detail previously [2]. Briefly, the probe was inserted into the channel and gently advanced. While the outer sheath was held at the target in the subsegmental bronchus, the inner probe was advanced slowly into the peripheral airway until it contacts with the mucosal surface. It was maintained atraumatically in that position for 5-7 s, allowing the fibre rod to absorb an estimated $20 \mu \mathrm{l}$ of ELF. The inner probe was then carefully withdrawn into the outer sheath, and both were removed together. The wet inner probe was cut, placed in a tube of known weight, and stored at $-80^{\circ} \mathrm{C}$ until analysis. All samples were free of blood contamination. The procedure was performed in triplicate from the same sub-segmental bronchus.

\section{BAL Collection and Laboratory Processing}

BAL samples were obtained from areas of new or progressive densities or from the right middle lobe if no radiographic change was observed. The same segment was used as for the prior BMS sampling. BAL was performed by introducing up to $180 \mathrm{ml}$ of warm sterile $0.9 \%$ saline solution into a lung sub-segment and aspirating back after each aliquot.

\section{Measurements of Biochemical Constituents}

The assay method used for the cytokines was the same for the BMS and BAL samples. Cytokine levels for IL-1 $\beta$, IL-6, IL-8, TNF- $\alpha$ and G-CSF were determined by addressable bead laser immunoassay. Further information on sample processing is available in an online supplementary file (see www.karger.com/ doi/10.1159/000368367).

\section{Statistical Analysis}

Patient data were presented descriptively for all 5 cohorts. Descriptive statistics data median 25 th and 75 th percentiles were used for the variables of ELF and BAL cytokine concentrations. In all 5 patient cohorts, data were assessed using SPSS v19 software (SPSS
Inc., Chicago, Ill., USA) and GraphPad Prism (GraphPad software, San Diego, Calif., USA). Parametric data was assessed using the Student $\mathrm{t}$ test with the Bonferroni correction where applicable. Non-parametric data were assessed using the Mann-Whitney U test and the Kruskal-Wallis test with the Dunn post hoc correction for multiple analyses. A $p$ value of $<0.01$ was considered significant, given the number of comparisons made.

\section{Results}

All bronchoscopic sampling (BMS and BAL) was well tolerated by all subjects, without any predefined adverse events. Anthropometric data are shown in table 1. Twelve patients were currently smoking at the time of recruitment and 12 were ex-smokers. Five patients had positive microbiology after bronchoscopic sampling, 4 with lower respiratory tract pathogens being isolated (no mycobacterial disease) and 1 with a urinary tract infection (known source prior to sampling of the patient with ALI). None of the non-ventilated patients with positive bronchial microbiology had clinico-pathological evidence of active infection. All cytokine concentrations were higher in the mechanically ventilated patients with ALI and burns-related inhalational injury than in those with stable chronic lung disease (table 2).

\section{BMS Probe}

The mean volume of ELF obtained from the BMS was $17 \mu \mathrm{l}$ (range 12-24 $\mu \mathrm{l}$ ). Concentrations of IL-1, IL-6, IL-8 and TNF- $\alpha$ were similar in the COPD and ILD groups, and not statistically different from the control group 


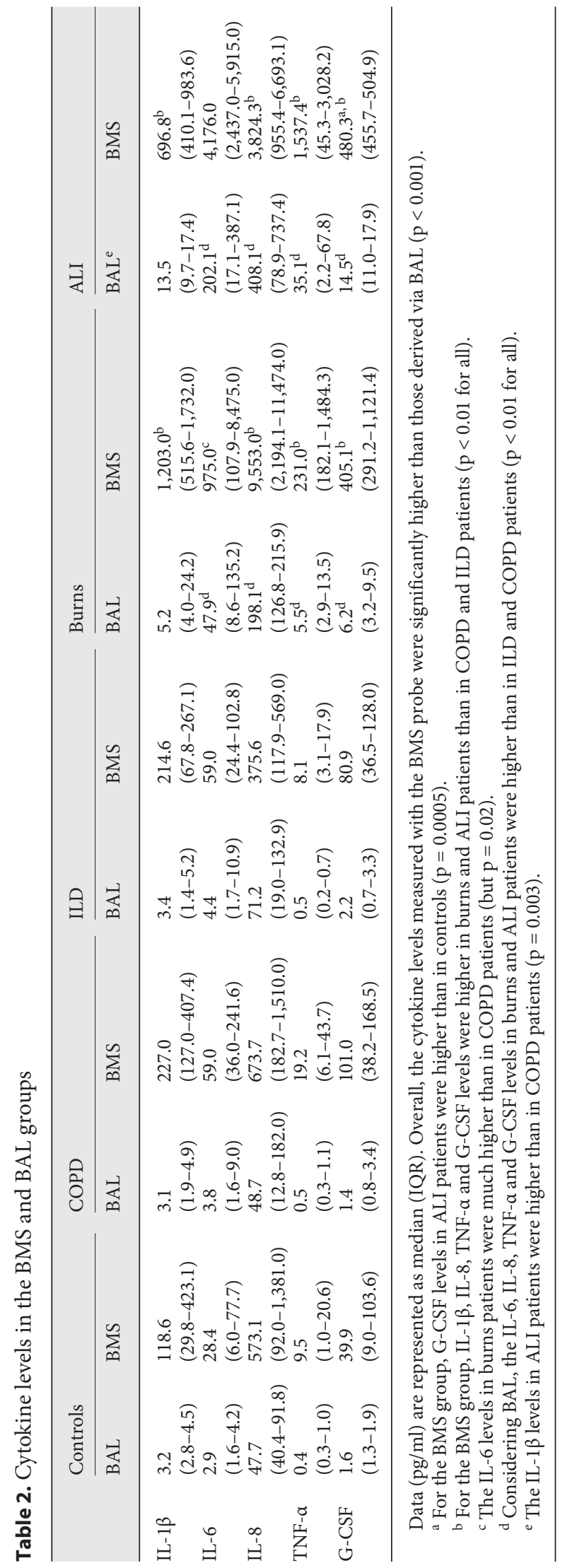

(fig. 1a-e). The concentration of G-CSF was significantly higher in the ALI group than in the other groups ( $\mathrm{p}=$ 0.0005). Concentrations of all cytokines were higher in the burns and ALI groups than in the COPD and ILD groups $(\mathrm{p}<0.01)$.

\section{Bronchoalveolar Lavage}

The mean BAL volume retrieved was $61 \pm 36 \mathrm{ml}$. The range used for analysis was $30-123 \mathrm{ml}$. The concentration of IL- $1 \beta$ was significantly higher in the burns and ALI groups than in the COPD and ILD groups $(\mathrm{p}<0.001)$. There was no statistical difference between the COPD, ILD and control groups.

The concentrations of IL-6, IL-8, TNF- $\alpha$ and G-CSF were higher $(p<0.01)$ in the burns group than in the control group (fig. 1b-e). The levels of IL-6, TNF- $\alpha$ and GCSF were higher in the burns and ALI groups than in the COPD and ILD groups ( $p<0.01$ for all). The level of IL-8 was significantly higher in the ALI group than in the ILD cohort $(\mathrm{p}=0.003)$.

\section{Comparison of BMS and BAL}

The concentrations of cytokines measured with the BMS probe were significantly higher than those measured in the BAL fluid $(\mathrm{p}<0.001)$. The inter-quartile range (IQR) of these concentrations was higher for the BMS than for the BAL sample (fig. 1a-e). The measured cytokine concentration ratios of BMS:BAL were examined for each pair of samples. For each patient group, there was no significant difference between the ratios as the confidence intervals were large (fig. 2a).

The effect of the patient cohort group on the BMS:BAL concentration of each cytokine was examined (fig. 3a-e). The ratios for the cytokines in the burns cohort (in particular for IL-1 $\beta$ ) were amongst the highest in the study, but no other discernable differences were identified.

\section{Discussion}

A prospective, observational, cohort study was undertaken on patients with chronic lung diseases, critically ill general and burns patients and those without chronic lung disease to determine whether sampling of ELF from the distal airways using a prototype BMS probe provided the same qualitative and quantitative information on the cytokines IL-1 $\beta$, IL-6, IL-8, TNF- $\alpha$ and G-CSF as with conventional BAL. The BMS probe was well tolerated, as was BAL. Both procedures were performed without complications. This reflects previous experience with BMS for obtaining 


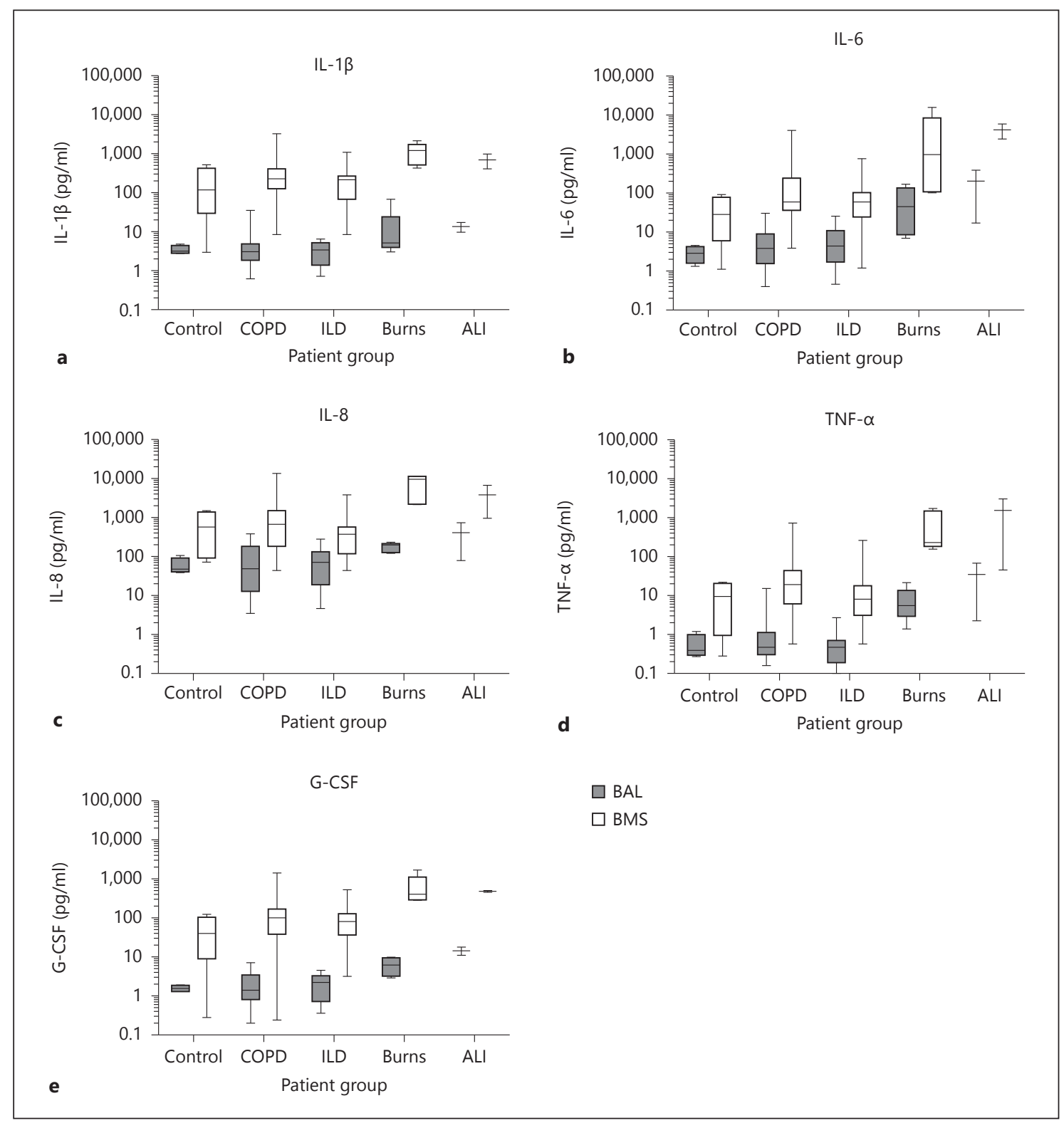

Fig. 1. Cytokine levels obtained from the BMS probe and via BAL. The charts demonstrate the levels of IL-1 $\beta$ (a), IL-6 (b), IL-8 (c), TNF- $\alpha(\mathbf{d})$ and G-CSF (e) in controls, patients with COPD and ILD and ICU patients with burns and ALI. The data are represented by box (median and IQR) and whisker (range) plots. Considering the BMS probe, the levels of G-CSF in the ALI group were higher than in the control group $(p=0.0005)$. For IL- $1 \beta$, IL-8, TNF- $\alpha$ and GCSF, cytokine levels were higher in the burns and ALI groups than

ELF to measure inflammatory cytokines in ventilated patients following cardiopulmonary bypass [10]. The BMS probe's retrieval of ELF demonstrated a hierarchy of cytokine levels according to the severity of the inflammatory illness. Patients with acute lung disease (burns-related in- in the COPD and ILD groups ( $\mathrm{p}<0.01$ for all). With IL-6, levels were higher in the burns group than in the COPD group (but $\mathrm{p}=0.02$ ). For the BAL study, IL-6, IL-8, TNF- $\alpha$ and G-CSF were higher in the burns and ALI groups than in the ILD and COPD cohorts $(\mathrm{p}<0.01$ for all). For IL-1 $\beta$, the levels in the ALI group were only significantly higher than in the COPD cohort $(\mathrm{p}=0.003)$. Overall, the cytokine levels measured from the BMS were significantly higher than those from the BAL $(p<0.001)$.

halational injury and ALI) produced higher cytokine levels than those with chronic stable lung disease (COPD and ILD), which were higher than from the controls. The cytokine levels in the chronic lung disease groups was not significantly different statistically to the controls; this may be 


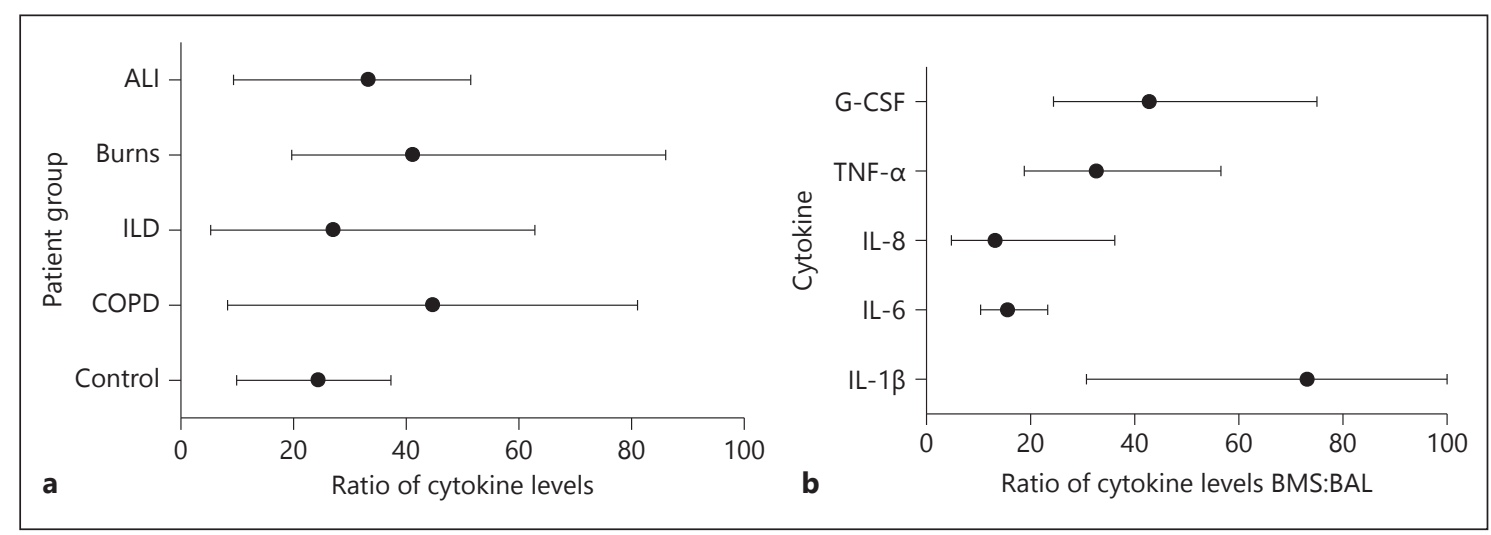

Fig. 2. Ratio of cytokine concentrations obtained from the BMS and by BAL according to patient group (a) and cytokine (b). Data are represented as means (95\% confidence interval). a There was no significant difference between the ratios per patient group at the

a reflection of the small number of patients in the latter group or be due to the fact that 3 out of the 4 healthy control patients had an active or previous history of smoking, which would have altered cytokine expression [11].

The ELF collection with the BMS probe was effective. This was evident from the concentrations of the cytokines, which were consistently higher than those sampled by BAL; this was the case for all cytokines and for the different patient groups (fig. 2a, b). It would appear that the cytokine concentrations from the BMS ELF were between 20 and 80 times greater than those from the non-ureacorrected BAL, depending on the patient group. One study determined the pulmonary levels of levofloxacin to be twice as high in the BAL group as in the BMS group [4]. If BAL levels were corrected using urea, an estimated 80 -fold dilution correction to BAL (obtained from a different patient cohort) would result in the BAL levels approximating to the BMS levels.

The greatest difference and highest concentrations of all cytokines were noted in the burns and ALI patients. This was to be expected in view of these patients having the greatest acute inflammatory response of all groups at the time of the bronchoscopic sampling.

No correlation between BMS and BAL for cytokine concentrations was established. The small group numbers are a study limitation that may have accounted for this. Alternatively, it may be that BMS ELF sampling is from a different (more proximal) part of lung, i.e. only the bronchiolar conducting airways, as opposed to the distal bronchioalveolar lining for BAL. If the cytokine milieu of each compartment differed, then the BMS/BAL ratio would vary according to the cytokine profile but not be-
$5 \%$ significance level. $\mathbf{b}$ The ratio for IL- $1 \beta$ was higher than for IL-6 $(\mathrm{p}=0.015)$ and IL-8 $(\mathrm{p}=0.03)$. The ratio for $\mathrm{G}-\mathrm{CSF}$ was higher than for IL-6 ( $\mathrm{p}=0.008$; Kruskal-Wallis test with Dunn post hoc correction).

tween patient groups. This is in keeping with the signal ratios seen in the study (fig. 2). The dilution of the sampled cytokines within the saline component of the BAL could also have contributed to the reduced concentrations when compared to the ELF samples obtained with the BMS probe. Although urea measurement could have been used to standardize BAL concentrations, this is not universally accepted. Dilutional errors may remain despite correction, contributing to the differences seen. Overall, however, it is likely that the BMS is from airways proximal to the alveoli. This needs to be borne in mind when interpreting the concentration levels. This may be an advantage in the case of burns patients, where smoke inhalation causes greater injury proximally. Indeed, the use of BMS may be a unique way of simultaneously sampling small and diverse areas of burns-injured and noninjured lung compared with the global measurement obtained with BAL. We believe that this is an important aspect for further study.

Collection of ELF by synthetic absorptive polyester matrices applied to the nasal mucosa has been described for cytokine analysis in allergic rhinitis. The low biomolecular binding, its stable retention of protein-containing constituents and highly hydrophilic absorbability are properties that may allow sampling of relatively undisturbed mucosal lining compared to lavage [12].

A number of conclusions can be drawn from this study. First, the BMS probe is an easy-to-use sampling device for measuring inflammatory cytokines from the distal airways through the working channel of a conventional bronchoscope. Second, BMS consistently identifies the same cytokines from acute and chronic lung disease 


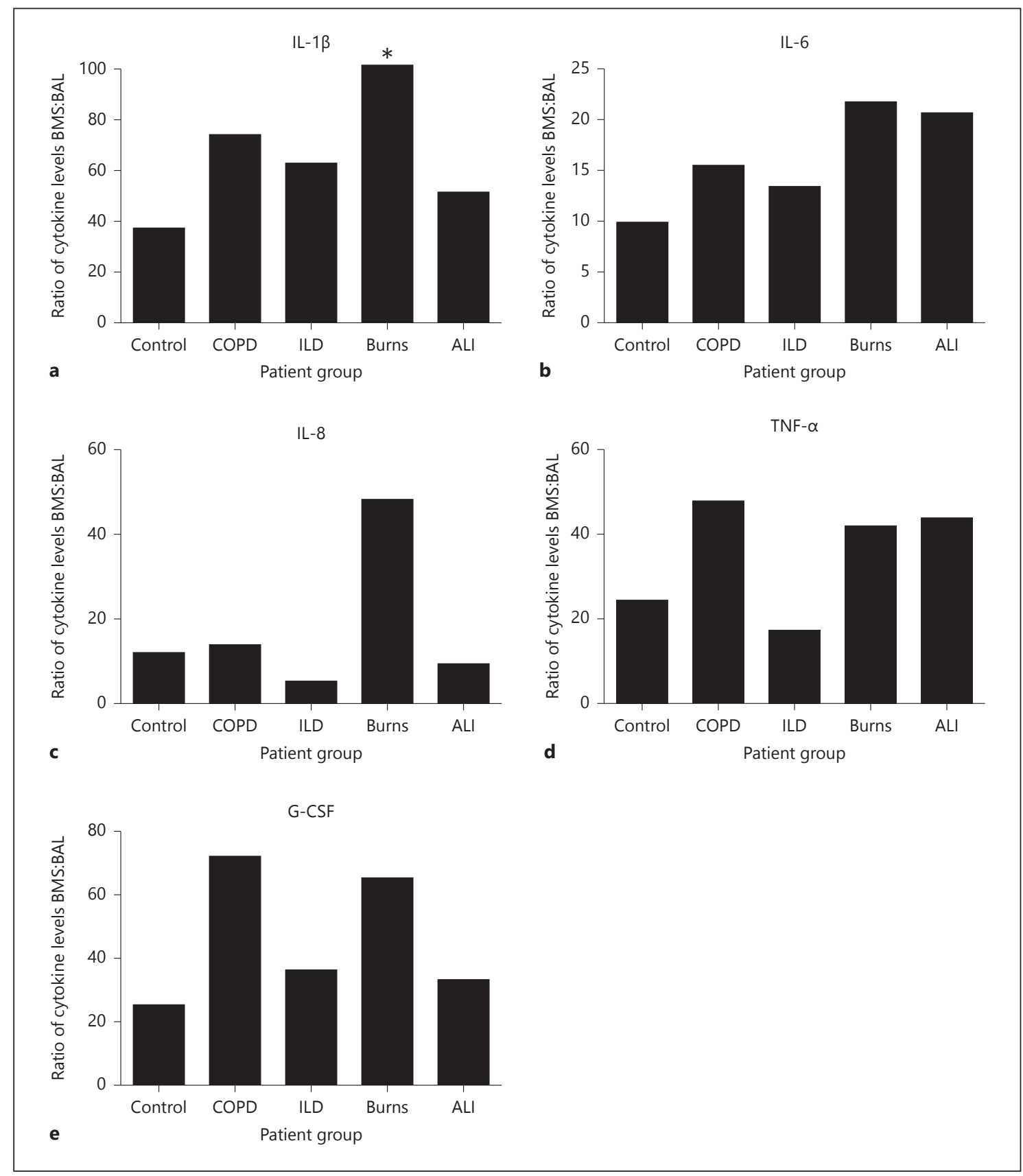

Fig. 3. The BMS:BAL cytokine ratios for the pooled patients in the 5 groups (healthy controls and patients with COPD, ILD, burns and ALI) are shown. The ratios have been subdivided by cytokine: IL-1 $\beta$ (a), IL-6 (b), IL-8 (c), TNF- $\alpha$ (d) and G-CSF (e). For IL-1 $\beta$, the ratio for burns was approximately 200, i.e. above the upper range of the graph. Overall, the ratios of BMS:BAL cytokines in the burns patients were amongst the highest but no other discernable differences were seen. ${ }^{*} \mathrm{p}<0.01$. as traditional BAL sampling, suggesting its potential as an alternative to BAL. This may be advantageous in settings where patients are at risk of hypoxia and would not tolerate saline lavage. Third, BMS provides sufficient ELF for measuring higher concentrations of inflammatory cyto- kines than can be provided by BAL. Fourth, there was a higher IQR per cytokine concentration for ELF from the BMS than from the BAL sample. Further study is necessary to understand whether sampling refinements are required. Finally, bronchoscopic microsampling is a useful 
tool to research cytokine levels in critically ill burns patients with inhalational injuries where proximal sampling with BAL would be difficult.

In summary, this study has shown the safety and potential utility of a BMS probe for biomarker sampling from bronchial ELF in patients with acute and chronic lung disease. There is at least the equivalent and likely an increase in the cytokine concentrations from ELF obtained by BMS probe compared to that obtained with BAL. Differences in cytokine measurements between the two methods suggest that the BMS method measures a cytokine milieu proximal to the more distal airways sampled by BAL.

\section{Key Messages}

(1) The BMS probe identifies similar cytokines to BAL in patients with both acute and chronic lung disease.

(2) There is a 20- to 80-fold increase in the concentration of cytokines measured with the BMS probe compared to via BAL.

(3) Differences in the concentrations of cytokines obtained from the ELF with these two methods may reflect the technical sampling differences of adsorption versus lavage or else be sampling-site-specific.
(4) The BMS probe may be a useful modality to study the inflammatory differences on proximal and distal airways in burns-related inhalational injury.

\section{Acknowledgements}

We are grateful to the patients and relatives who consented to recruitment into this study. We recognise the invaluable support of staff of the bronchoscopy unit, ICU and burns unit at the Chelsea and Westminster Hospital. The BMS probes were kindly provided by Olympus, Tokyo, Japan. We acknowledge the work of the Immunology section, Imperial College, London under the guidance of Dr. Peter Charles and Dr. Peter Kelleher. Finally, we are most grateful to the joint research committee of the Westminster Medical School Research Trust for the award of a small grant enabling the work to be carried out.

\section{Financial Disclosure and Conflicts of Interest}

Olympus provided the BMS microprobes for this study. It had no input or influence on any part of the study or its analysis and interpretation.

\section{References}

1 Rennard SI, Basset G, Lecossier D, O’Donnell KM, Pinkston P, Martin PG, Crystal G: Estimation of volume of epithelial lining fluid recovered by lavage using urea as marker of dilution. J Appl Physiol 1985;60:532-538.

-2 Ishizaka A, Watanabe M, Yamashita T, Ogawa $\mathrm{Y}$, Koh H, Hasegawa N, Nakamura H, Asano K, Yamaguchi K, Kotani M, Kotani T, Morisaki H, Takeda J, Kobayashi K, Ogawa S: New bronchoscopic microsample probe to measure the biochemical constituents in epithelial lining fluid of patients with acute respiratory distress syndrome. Crit Care Med 2001;29:896-898.

3 Ishizaka A, Matsuda T, Albertine KH, Koh H, Tasaka S, Hasegawa N, Kohno N, Kotani T, Morisaki H, Takeda J, Nakamura M, Fang X, Martin TR, Matthay MA, Hashimoto S: Elevation of KL-6, a lung epithelial cell marker, in plasma and epithelial lining fluid in acute respiratory distress syndrome. Am J Phystiol Lung Cell Mol Physiol 2004;286:L1088L1094.
4 Yamazaki K, Ogura S, Ishizaka A, Oh-hara T, Nishimura M: Bronchoscopic microsampling method for measuring drug concentration in epithelial lining fluid. Am J Respir Crit Care Med 2003;168:1304-1307.

5 Kikuchi J, Yamazaki K, Kikuchi E, Ishizaka A, Nishimura M: Pharmacokinetics of telithromycin using bronchoscopic microsampling after single and multiple oral doses. Pulm Pharm Ther 2007;20:549-555.

-6 Watanabe M, Ishizaka A, Ikeda E, Ohashi A, Kobayashi K: Contributions of bronchoscopic microsampling in the supplemental diagnosis of small peripheral lung carcinoma. Ann Thorac Surg 2003;76:1668-1673.

7 Bernard GR, Artigas A, Brigham KL, Carlet J, Falke K, Hudson L, Lamy M, LeGall JR, Morris A, Spragg R: Report of the AmericanEuropean Consensus Conference on acute respiratory distress syndrome: definitions, mechanisms, relevant outcomes, and clinical trial coordination. Consensus Committee. J Crit Care 1994;9:72-81.

-8 Endorf FW, Gamelli RL: Inhalational injury, pulmonary perturbations, and fluid resuscitation. J Burn Care Res 2007;28:80-83.
-9 Vestbo J, Hurd SS, Agusti AG, Jones PW, Vogelmeier C, Anzueto A, Barnes PJ, Fabbri LM, Martinez FJ, Nishimura M, Stockley RA, Sin DD, Rodriguez-Roisin R: Global strategy for the diagnosis, management, and prevention of chronic obstructive pulmonary disease: GOLD executive summary. Am J Respir Crit Care Med 2013;187:347-365.

10 Kotani T, Kotake Y, Morisaki H, Takeda J, Shimizu H, Ueda T, Ishizaka A: Activation of a neutrophil-derived inflammatory response in the airways during cardiopulmonary bypass. Anesth Analg 2006;103:1394-1399.

-11 McCrea KA, Ensor JE, Nall K, Bleecker ER, Hasday JD: Altered cytokine regulation in the lungs of cigarette smokers. Am J Respir Crit Care Med 1994;150:696-703.

-12 Chawes BL, Edwards MJ, Shamji B, Walker C, Nicholson GC, Tan AJ, Følsgaard NV, Bønnelykke K, Bisgaard H, Hansel TT: A novel method for assessing unchallenged levels of mediators in nasal epithelial lining fluid. J Allergy Clin Immunol 2010;125:1387-1389. 